\title{
Massifs de sol renforcés par inclusions rigides : analyse de l'effet d'un chargement cyclique vertical quasi statique par expérimentation sur modèle réduit et validation numérique
}

\author{
Moustafa Houda ${ }^{1}$, Orianne Jenck ${ }^{2}$ et Fabrice Emeriault ${ }^{3, \star}$ \\ ${ }^{1}$ Laboratoire LOCIE, UMR 5271 USMB-CNRS, Université de Savoie, bâtiment HELIOS, 73376 Le Bourget-du-Lac cedex, France \\ 2 Laboratoire 3SR, UMR 5521 CNRS, Université Grenoble-Alpes, 38041 Grenoble cedex 9, France \\ ${ }^{3}$ Laboratoire 3SR, UMR 5521 CNRS, Université Grenoble-Alpes, 38041 Grenoble cedex 9, France
}

\begin{abstract}
Résumé - Les recommandations publiées par le projet national français d'amélioration du sol par inclusions rigides (ASIRI) ont été limitées aux cas de chargement monotone. Cependant, différents cas de structures sous chargement cyclique sont couramment rencontrés dans la pratique, ce qui nécessite de comprendre le comportement sous chargement cyclique et/ou dynamique de ces ouvrages. Le travail de recherche présenté dans cet article constitue une contribution à la compréhension du comportement de cette technique lors d'un chargement cyclique vertical quasi statique. La première étape du travail a consisté en la réalisation d'une campagne d'essais sur un modèle réduit en laboratoire simulant le cas d'inclusions rigides avec ou sans dallage en surface et mettant en œuvre une méthode de corrélation d'images. La modélisation physique nous a permis d'étudier l'influence de la présence du dallage à la surface de la plateforme de transfert de charge (PTC) et du préchargement sur le comportement de cette technique sous chargement cyclique. Les résultats expérimentaux montrent une accumulation des tassements durant le chargement cyclique quelle que soit la condition limite appliquée en surface. Ils montrent également que la présence du dallage augmente l'accumulation observée durant les cycles. En revanche, elle conduit à une réduction du tassement total lors d'un chargement monotone ultérieur. Dans un deuxième temps, les résultats expérimentaux ont été utilisés comme une base de données pour la validation d'un modèle numérique en milieu continu avec le logiciel FLAC ${ }^{3 \mathrm{D}}$.
\end{abstract}

Mots clés : amélioration de sol / inclusions rigides / plateforme de transfert de charge / chargement cyclique / modélisation physique / corrélation d'images / modélisation numérique

\begin{abstract}
Soil reinforcement by rigid piles: analysis of the influence of vertical cyclic quasi-static loading by experimentation on small-scale model and numerical validation. The recommendations established by the French National Project on soil reinforcement by rigid inclusions (ASIRI) were limited to the case of monotonic loading. Yet, various cases of structures under cyclic loading are commonly encountered in the practice, which requires the understanding of this technique under cyclic and/or dynamic loading. The research work presented in this paper is a contribution to the understanding of the behaviour of this type of structures under a quasi-static vertical cyclic loading. The first step of the study consists in performing a series of tests on a small-scale laboratory model simulating the case of rigid inclusions with or without rigid slab at the ground surface and implementing a digital image correlation method. The parametric study performed with the physical modeling allowed us to study the influence of the presence of a rigid slab at the surface of the load transfer platform (LTP) and of the preloading on the behaviour of this technique under cyclic loading. The experimental results highlighted an accumulation of the settlements during the cyclic loading in both cases: with and without rigid slab. They also showed that the presence of the rigid slab increases the settlements' accumulation observed during the cycles. Nevertheless, the presence of the slab results in a reduction of the total settlement during a further monotonic loading. In a second step, the experimental results have served as a database for the validation of a numerical model using the software FLAC ${ }^{3 \mathrm{D}}$.
\end{abstract}

Keywords: soil reinforcement / rigid inclusions / load transfer platform / cyclic loading / physical modeling / digital image correlation / numerical modeling

* Auteur correspondant : fabrice.emeriault@3sr-grenoble.fr 


\section{Introduction}

Le besoin croissant pour le développement d'infrastructures amène souvent les ingénieurs à concevoir et dimensionner des projets de construction sur des sols compressibles. L'application d'un chargement externe à la surface du sol compressible introduit des grandes déformations totales et différentielles. Ces déformations doivent être limitées pour maintenir la stabilité, la durabilité et le bon fonctionnement des ouvrages concernés. Parmi les différentes méthodes de renforcement de sols compressibles, la technique d'amélioration par inclusions rigides verticales s'est particulièrement développée depuis plusieurs années. Elle constitue une alternative intéressante aux techniques plus traditionnelles et un bon compromis entre la réduction des tassements effectifs et celle des coûts de construction (Magnan, 1994). Cette technique de renforcement est différente de la technique classique de fondation sur pieux car les inclusions rigides ne sont pas reliées directement à la superstructure : le massif de sol renforcé par inclusions est recouvert d'une couche de matériau granulaire appelée «plateforme de transfert de charge » ou PTC. Dans cette technique de renforcement de sols, il faut distinguer deux types d'ouvrage :

- les ouvrages de type "dallage », pour lesquels une dalle rigide est intercalée entre la PTC et la superstructure, ce qui introduit une répartition sensiblement uniforme des tassements et donc une répartition de contrainte non uniforme en surface de la PTC ;

- les ouvrages de type « remblai », qui introduisent une répartition quasi uniforme de la contrainte en surface de la PTC et donc une répartition non uniforme des tassements de celle-ci.

La majorité des études expérimentales existantes pour l'étude du comportement de cette technique s'intéressent au cas de chargement monotone. Néanmoins, ces études ont permis de mettre en évidence les mécanismes de report de charge dans le matelas ainsi qu'un certain nombre de facteurs affectant ces mécanismes. Certaines de ces études ont été menées avec des modèles physiques réduits bidimensionnels (De Pasquale, 2011 ; Antoine, 2010 ; Chen et al., 2008 ; Jenck, 2005 ; Horgan et Sarsby, 2002). Des expérimentations sur modèles réduits tridimensionnels ont également été menées par Van Eekelen et al. (2012a, 2012b), Eskisar et al. (2012), Dinh (2010), Kempfert et al. (1997) et Demerdash (1996). Un certain nombre de recherches ont été effectuées en centrifugeuse dont la plupart ont été réalisées sur des modèles réduits tridimensionnels (Okyay et al., 2014 ; Baudouin, 2010 ; Ellis et Aslam, 2009a, 2009b). D'autres travaux ont été réalisés dans le but de visualiser les mécanismes prenant place à l'intérieur de la PTC. La plupart de ces travaux ont été réalisés sur des modèles réduits bidimensionnels. Certains de ces modèles ont suivi la déformation du remblai à travers une vitre latérale en colorant les différentes couches du remblai (Chen et al., 2008 ; Horgan et Sarsby, 2002 ; Low et al., 1994 ; Hewlett et Randolph, 1988), et quelques autres ont mesuré les champs de déplacement à l'intérieur de la PTC par la méthode de corrélation d'images numériques (DIC) (Jenck et al., 2014 ; Antoine, 2010 ; Jenck et al., 2007).

Sous chargement cyclique, les cas de charges variables répétées avec un très grand nombre de cycles de type charge de trafic sont plus largement étudiés (Heitz et al., 2008 ; Van Eekelen et al., 2007 ; Heitz, 2006) que le cas du chargement cyclique de type réservoir de stockage (Briançon et al., 2015 ; Okyay et al., 2014 ; Morgon, 2010 ; Rault et Thorel, 2009 ; Dinh, 2010).

La bibliographie, même récente, montre que les études numériques portant sur le comportement de massifs de sol renforcés par inclusions rigides se limitent, en général, au cas d'un chargement monotone, qu'il s'agisse de modélisations numériques en milieu continu (Briançon et al., 2015 ; Okyay et Briançon, 2012 ; Okyay et Dias, 2010 ; Jenck et al., 2009 ; Huang et Han, 2009 ; Laurent et al., 2003 ; Han et Gabr, 2002) ou en milieu discret (Han et al., 2011 ; Chevalier et al., 2011).

Sous chargement cyclique, les travaux numériques publiés à ce jour s'intéressent plus particulièrement au cas de chargement répété de type trafic routier ou ferroviaire, traités par éléments finis (Heitz, 2006 ; Arwanitaki et Triantafyllidis, 2006) ou par éléments discrets (Han et Bhandari, 2009 ; Villard et Grange, 2013), ou étudient le comportement des ouvrages renforcés par inclusions rigides sous sollicitations sismiques (Thai Son et al., 2010 ; Santruckova, 2012).

Cet article présente une étude expérimentale sur un modèle physique réduit, suivie d'une validation numérique, du cas des chargements cycliques verticaux d'amplitude relativement importante et de fréquence faible pour des matelas de faible épaisseur, avec ou sans dallage, supportant un réservoir de stockage, une grue, etc.

\section{Dispositif expérimental}

Un modèle physique tridimensionnel à une échelle réduite de $1 / 10^{\mathrm{e}}$ sur les longueurs et sous gravité normale a été conçu et développé au sein du laboratoire 3SR (Fig. 1). Son objectif est d'étudier l'efficacité et la pérennité de la technique d'amélioration des sols par inclusions rigides sous chargement cyclique. Le modèle consiste en une cuve métallique de dimensions internes en plan $1 \times 1 \mathrm{~m}$, à l'intérieur de laquelle 16 inclusions rigides en aluminium de diamètre $d=0,035 \mathrm{~m}$ sont fixées sur le fond du modèle et réparties suivant un maillage carré, avec un espacement entre inclusions $s=0,2 \mathrm{~m}$. Quatre demi-inclusions sont ajoutées contre une vitre transparente latérale de $435 \times 335 \mathrm{~mm}$, ce qui permet la visualisation depuis l'extérieur des mécanismes qui se développent dans le matelas de transfert de charge durant les différentes étapes de chargement. Le taux de recouvrement $\alpha$ dans la zone centrale est ainsi de $2,4 \%$, ce qui correspond à une valeur classiquement rencontrée sur ouvrage réel en France. Pour la campagne expérimentale présentée dans cet article, l'épaisseur de sol compressible est fixée et égale à $0,4 \mathrm{~m}$ alors que l'épaisseur $\mathrm{H}$ de la PTC a été fixée égale à $0,1 \mathrm{~m}$ (Fig. 2). Une surcharge uniformément répartie en surface est appliquée par une membrane sous pression d'eau. Il est également possible de réaliser des essais avec des conditions aux limites différentes en ajoutant une dalle rigide à la surface de la PTC imposant par suite des déplacements uniformes en surface.

Le sol compressible est simulé par un mélange de billes de polystyrène expansé et de sable de Fontainebleau (à une teneur en eau de $10 \%$ pour éviter la ségrégation lors de la mise en place du mélange dans la cuve). Ce type de mélange a été utilisé dans des essais en laboratoire au cours du PN ASIRI (Dinh, 

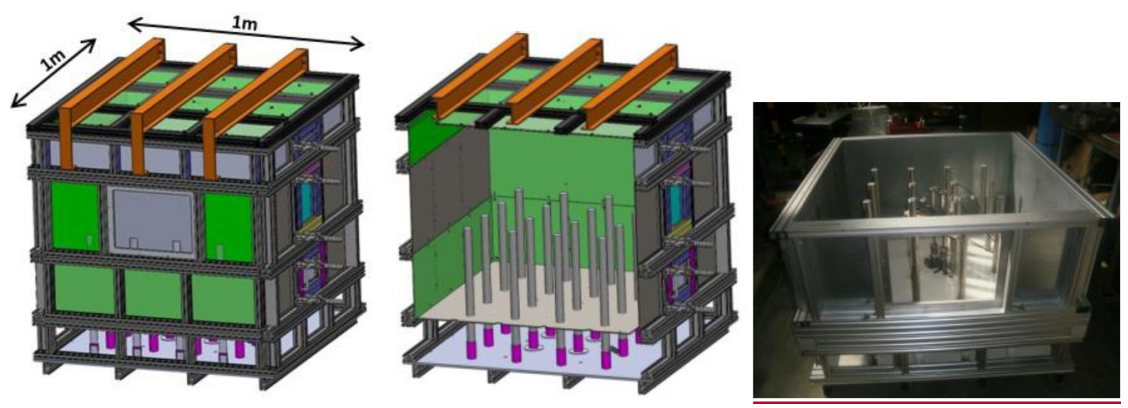

Fig. 1. Vue schématique en 3D du modèle et photographie.

Fig. 1. 3D schematic view of the model and picture.

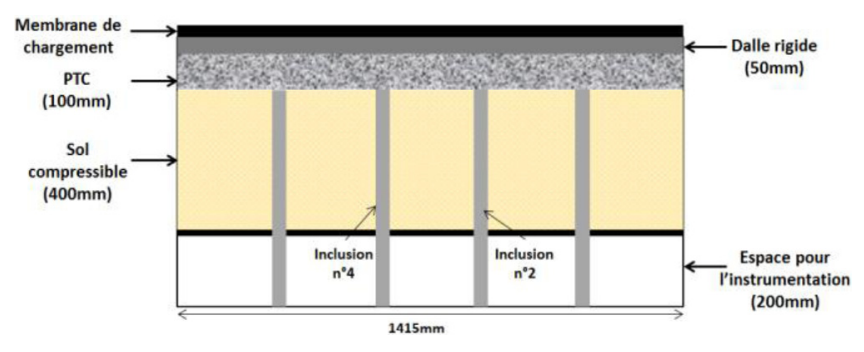

Fig. 2. Coupe schématique du dispositif.

Fig. 2. Schematic section of the model.

Tableau 1. Caractéristiques de compressibilité du sol.

Table 1. Compressibility characteristics of the soil.

Indice de compression $\mathrm{Cc}$

Indice de gonflement $\mathrm{Cs}$

Indice des vides initial $\mathrm{e}_{0}$

$\mathrm{Cc} /\left(1+e_{0}\right)$

Pression de préconsolidation $\sigma / \mathrm{p}$

Tableau 2. Caractéristiques du gravillon constituant la plateforme de transfert de charge (PTC).

Table 2. Characteristics of gravel constituting the load transfer platform (LTP).

\begin{tabular}{lll}
\hline Angle de frottement pic & $\Phi /$ pic & $45^{\circ}$ \\
Angle de frottement palier & $\Phi /$ palier & $42^{\circ}$ \\
Cohésion & $c \prime$ & $0 \mathrm{kPa}$ \\
Dilatance & $\psi$ & $5^{\circ}$ \\
\hline
\end{tabular}

2010). Une campagne d'essais œdométriques a été réalisée sur des éprouvettes de $150 \mathrm{~mm}$ de diamètre afin de déterminer les caractéristiques de compressibilité de ce sol analogique, les résultats sont présentés dans le Tableau 1. En ce qui concerne la PTC, généralement constituée d'un sol granulaire noble, des gravillons concassés de diamètre $\emptyset \mathrm{g}$ compris entre 2 et $5 \mathrm{~mm}$ ont été utilisés. Cela représenterait un sol de granulométrie $20-50 \mathrm{~mm}$ à l'échelle du prototype. La réalisation d'essais triaxiaux monotones nous a permis de déterminer les caractéristiques de résistance au cisaillement de ce matériau (Tab. 2). Le comportement cyclique a été plus particulièrement

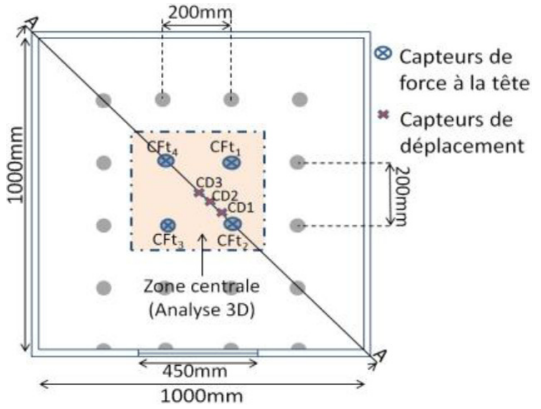

Fig. 3. Vue en plan du modèle avec instrumentation.

Fig. 3. Plan view of the model with instrumentation.

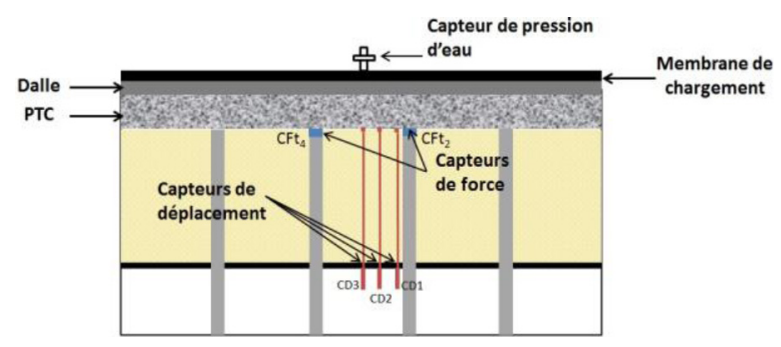

Fig. 4. Vue en coupe schématique A-A du modèle avec instrumentation.

Fig. 4. Schematic sectional view $A-A$ of the model with instrumentation.

apprécié par des essais triaxiaux cycliques dont les résultats sont utilisés dans la partie 5. La PTC est mise en place par couches successives de 0,025 m d'épaisseur, le matériau étant compacté à l'aide d'une dame spécifiquement conçue pour la campagne expérimentale. La densité obtenue après compactage est de $15,5 \mathrm{kN} / \mathrm{m}^{3}$ et correspond à une densité relative $\mathrm{I}_{\mathrm{D}}=75 \%$.

L'analyse des interactions sol-structure, complexes qui se développent dans le modèle, nécessite une instrumentation idoine (Fig. 3 et 4 ). Ont ainsi été mesurés dans la maille centrale :

- les tassements à l'interface sol compressible/PTC en trois points (notés D1, D2 et D3) à l'aide de trois capteurs de déplacement potentiométriques ;

- les forces en tête des quatre inclusions centrales (notées $\mathrm{Ft}_{1}$, $\mathrm{Ft}_{2}, \mathrm{Ft}_{3}$ et $\mathrm{Ft}_{4}$ ) via quatre capteurs de force. 
Tableau 3. Programme des essais.

Table 3. Programme of tests.

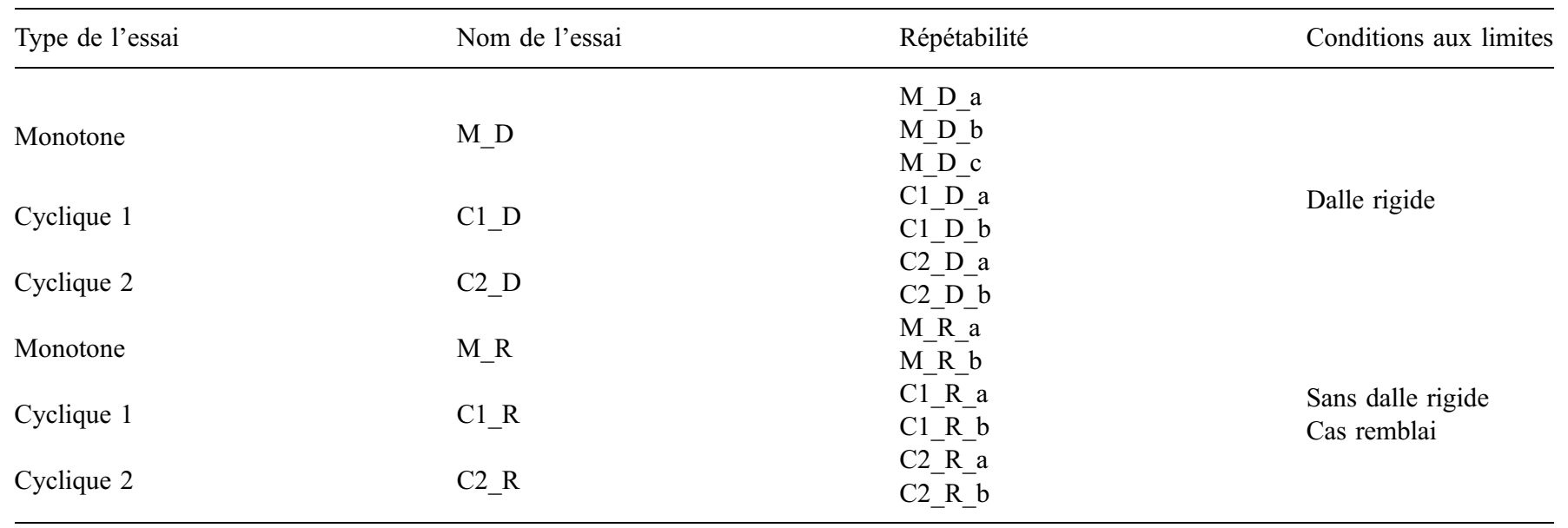

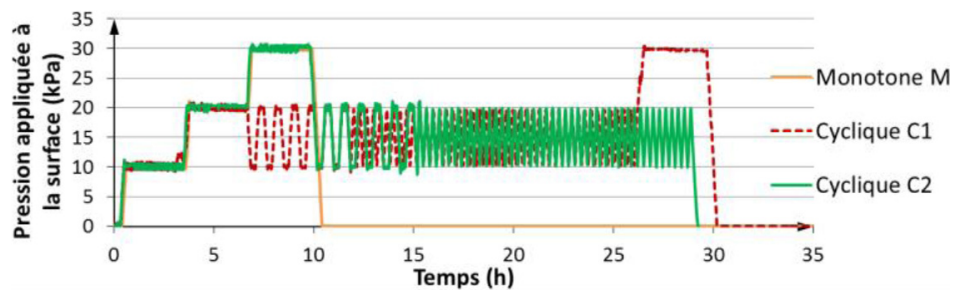

Fig. 5. Types de chargements appliqués.

Fig. 5. Types of applied loads.

Ce dispositif permet d'étudier les mécanismes en zone centrale autour des quatre inclusions centrales, en s'affranchissant au maximum des effets de bord.

Une analyse du champ de déplacement pendant les chargements monotone et cyclique a été également réalisée afin d'avoir une meilleure compréhension de la cinématique et des mécanismes développés dans le matelas granulaire de transfert de charge. La technique de "Particle Image Tracking » (PIT) basée sur la méthode de DIC (Houda et al., 2016) est utilisée pour déterminer le mouvement de corps rigide en $2 \mathrm{D}$ des grains constituant le matériau de la PTC. L'outil de calcul utilisé est le code «Tracker » conçu au laboratoire 3SR par Combe et Richefeu (2013).

Les avantages du dispositif expérimental sont :

- des essais rapides à mettre en œuvre ;

- la prise en compte de la compressibilité du sol à renforcer par les inclusions avec un temps de « consolidation » très faible comparé à la réalité ;

- une exploitation du champ de déplacement latéral (mais qui reste qualitatif car possiblement affecté par effets de bords sur la vitre sur laquelle aucune lubrification n'a été appliquée) ;

- des mesures précises car faites sur la partie centrale et les quatre inclusions qui la composent.

A contrario, les limitations du dispositif sont :

- une difficulté à vérifier l'absence d'effets de bords (même si une graisse est appliquée sur les frontières latérales pour limiter les frottements) ;
- des lois de similitude qui ne peuvent pas être totalement respectées (à l'inverse d'essais en centrifugeuse) ;

- un effet possible de la non-linéarité du comportement des sols (effet de pression).

Le Tableau 3 résume l'ensemble des essais réalisés sur le modèle physique et qui seront analysés dans le présent article. Deux conditions aux limites différentes ont été appliquées à la surface de la PTC. Un déplacement vertical uniforme est imposé à la surface de la PTC dans le cas des essais type (D) réalisés avec dallage. En revanche, pour les essais type (R) réalisés avec les conditions aux limites type remblai, c'est la répartition de la contrainte appliquée à la surface de la PTC qui est uniforme. Le programme des essais est défini de façon à évaluer l'effet des cycles, du préchargement du système avant l'application des cycles et de la présence de la dalle sur le comportement du système.

Le chargement monotone $(\mathrm{M})$ consiste à appliquer trois paliers de chargement de pression en surface de 10,20 et $30 \mathrm{kPa}$, suivis d'une décharge complète.

Deux types de chargement cyclique (Fig. 5) ont été appliqués :

- cyclique $1(\mathrm{C} 1)$ : l'essai consiste à appliquer deux paliers de chargement en surface de 10 et $20 \mathrm{kPa}$, suivis de 50 cycles entre 10 et $20 \mathrm{kPa}$ (cas des réservoirs et zones de stockage), suivis d'un chargement monotone à $30 \mathrm{kPa}$, puis d'une décharge jusqu'à $0 \mathrm{kPa}$;

- cyclique 2 (C2) : l'essai consiste à appliquer trois paliers de chargement de 10, 20 et $30 \mathrm{kPa}$ (préchargement du massif), 


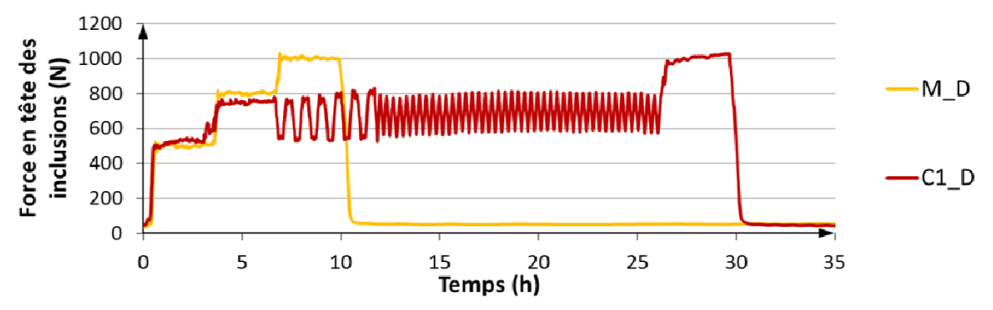

Fig. 6. Évolution de la force moyenne en tête des quatre inclusions centrales au cours des essais M_D et C1_D.

Fig. 6. Evolution of the average force at the head of four central inclusions for the tests $M_{-} D$ and $C 1 \_D$.

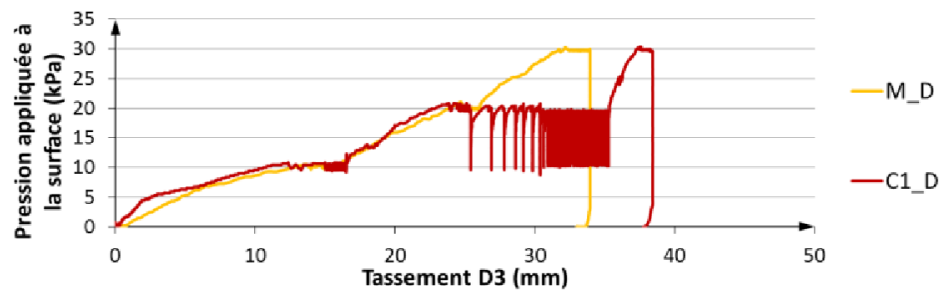

Fig. 7. Évolution du tassement D3 avec la pression appliquée à la surface pour les essais M_D et C1_D.

Fig. 7. Settlement evolution of $D 3$ with the applied pressure at the surface for the tests $M_{-} D$ and $C 1 \_D$.

suivis de 50 cycles entre 10 et $20 \mathrm{kPa}$, suivis d'une décharge finale jusqu'à $0 \mathrm{kPa}$.

Le chargement cyclique a été appliqué avec une période de 50 minutes pour les six premiers cycles, notamment afin de mettre en place l'analyse par imagerie, et de 20 minutes pour les 44 cycles suivants.

\section{3 Étude de l'influence du chargement cyclique avec dallage}

On s'attache ici à faire une première analyse détaillée de l'influence du chargement cyclique sur le comportement du système avec dallage. Les résultats de l'essai monotone M_D sont donc ici comparés avec ceux de l'essai cyclique C1_D. Les deux essais sont réalisés avec la même configuration géométrique.

La Figure 6 permet d'étudier l'impact du chargement cyclique sur le transfert de charge vers la tête des inclusions durant et après l'application des cycles. Pour l'essai C1_D et après l'application du chargement cyclique, pour une pression appliquée à la surface qui passe de 20 à $30 \mathrm{kPa}$, on retrouve les mêmes valeurs de la force moyenne en tête des inclusions que celles mesurées pour l'essai monotone M_D. Cela signifie que, pour la configuration étudiée, les mécanismes induits dans la PTC et le sol compressible par le chargement cyclique ne modifient pas de manière importante les transferts de charge vers la tête des inclusions et leur évolution suite à une augmentation de la pression en surface après le chargement cyclique. Cela est cohérent avec les observations faites par Morgon (2010) et par Okyay et al. (2014). La stabilité des efforts transmis aux inclusions avant et après les chargements cycliques associée aux déplacements importants mesurés (et confirmés par l'analyse d'image) est interprétée comme un mécanisme de poinçonnement progressif de la PTC par la tête des inclusions associé au développement en parallèle «d'extension» d'inclusions au travers de la PTC. Ce mécanisme est systématiquement observé.
Le tassement incrémental D3 présente une bonne répétabilité entre les deux essais $M \_D$ et $C 1 \_D$ pour les deux premiers paliers de chargement monotones (Fig. 7). L'application des cycles introduit pour l'essai C1_D une accumulation des tassements égale à $10 \mathrm{~mm}$ et qui se fait avec un rythme décroissant sans arriver pour autant à une stabilisation du tassement à la fin des 50 cycles. Durant les cycles, l'accumulation des tassements se fait lors des phases de rechargement et surtout pour des valeurs de pression autour de la valeur maximale de pression des cycles, entre 17 et $20 \mathrm{kPa}$. On n'observe en revanche aucune évolution du tassement durant les phases de déchargement. Le sol compressible analogique présente un léger phénomène de fluage. Cependant, pour une durée équivalente à celle du chargement cyclique, le tassement induit est égal à $1 \mathrm{~mm}$ sous $10 \mathrm{kPa}$ et $1,5 \mathrm{~mm}$ sous $20 \mathrm{kPa}$, ce qui représente au maximum $15 \%$ de la valeur totale du tassement accumulée durant les cycles de l'essai C1_D. Ceci permet de conclure que la majorité des déplacements observés sont liés au chargement cyclique stricto sensu.

La valeur du tassement mesurée à la fin du chargement cyclique dépasse celle de l'essai M_D pour le palier de chargement à $30 \mathrm{kPa}$. L'augmentation de la pression appliquée $P m$ de 20 à $30 \mathrm{kPa}$ après le chargement cyclique amène à un tassement final pour l'essai cyclique C1_D qui est $15 \%$ plus important que celui de l'essai monotone ${ }_{-}$_D. On observe en revanche que le déplacement incrémental pendant la phase de chargement de 20 à $30 \mathrm{kPa}$ est beaucoup plus faible après le chargement cyclique qu'en sollicitation monotone $(3 \mathrm{~mm}$ contre $8 \mathrm{~mm}$ pour le chargement monotone).

Ces résultats en matière de tassements sont tout à fait comparables aux conclusions de Heitz et al. (2008) qui ont réalisé des essais dans des conditions similaires (en particulier avec une condition aux limites de type dallage. On retiendra que l'accumulation de tassement lors des cycles peut mener à une valeur de tassement supérieure à celle obtenus sous chargement statique.

Pour mieux appréhender l'accumulation des tassements durant l'application des cycles, on peut utiliser une représentation 


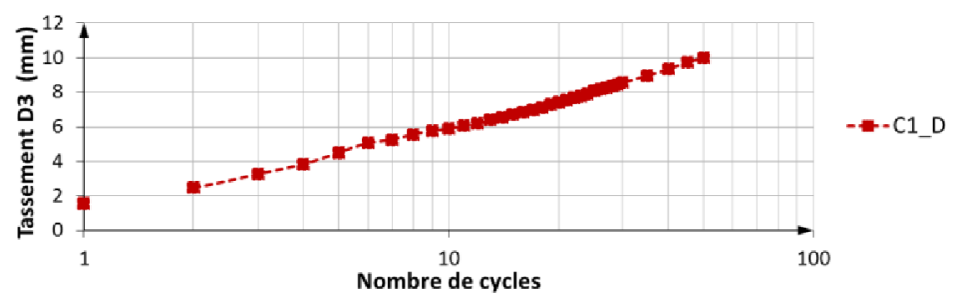

Fig. 8. Accumulation de tassement (D3) au pic des cycles en fonction du nombre de cycles pour l'essai C1_D.

Fig. 8. Settlement accumulation at the peak of the cycles versus of the number of cycles for the test C1_D.

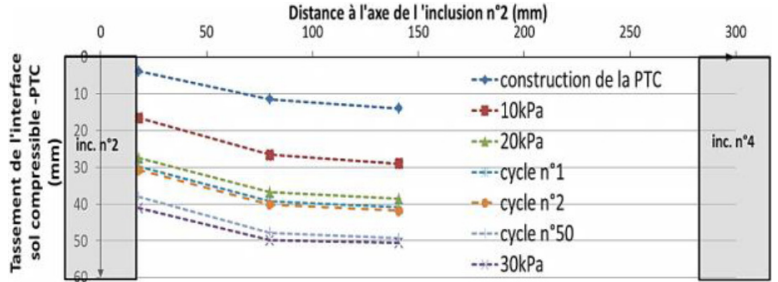

Fig. 9. Évolution du profil de tassement pour l'essai C1_D.

Fig. 9. Profil settlement evolution for the test C1_D.

des tassements au pic de chaque cycle (pour $P m=20 \mathrm{kPa}$ ) en fonction du logarithme du nombre de cycles (Fig. 8). Il apparaît que l'évolution est sensiblement linéaire, permettant ainsi une prévision de l'accumulation totale de tassement pour un nombre de cycles donné. Dans les conditions de l'essai, on retiendra qu'environ $60 \%$ de l'accumulation totale de tassement pendant le chargement cyclique se fait durant les dix premiers cycles.

À partir des trois capteurs de déplacement placés à la base de la PTC, il est possible de tracer le profil de tassement pour les différentes étapes de l'essai C1_D (Fig. 9). La forme du profil dû à la construction du modèle $n$ 'apparaît pas modifiée durant l'application du chargement cyclique.

La méthode de corrélation d'images a été appliquée sur l'essai C1_D afin de suivre l'évolution du champ de déplacements des grains dans la PTC durant les différentes étapes de chargement cyclique. La Figure 10 présente l'évolution du champ de déplacement vertical à la fin de chacun des six premiers cycles de l'essai C1_D. Chaque grain visible de la PTC est représenté par un polygone (cellule de Voronoii) et coloré suivant le déplacement vertical qui lui correspond. Lorsque que le critère de qualité de la corrélation d'image (zero-mean normalized cross-correlation [ZNCC] $>0,7)$ n'est pas rempli, la cellule est laissée blanche. De plus, sur la Figure 10, le niveau de la dalle rigide ainsi que la position des grains (cellules Voronoï) sont gardés fixes, dans la même position que celle de l'état de référence (fin du palier de chargement à $20 \mathrm{kPa}$ ). L'accumulation des tassements durant les six premiers cycles peut être observée clairement par le changement des couleurs des cellules en passant d'un cycle à un autre. Cette accumulation est plus importante dans la partie centrale, entre les deux inclusions, qu'au-dessus des inclusions. On met ainsi en évidence le développement des mécanismes de cisaillement dans le matelas granulaire de transfert de charge durant les cycles.

L'analyse du champ de déplacement vertical durant le premier cycle de déchargement-rechargement de l'essai C1_D (Fig. 11) montre clairement que, lors du déchargement, il n'y a presque pas d'évolution du champ de déplacement vertical, mais que c'est lors du rechargement et surtout entre 17 et

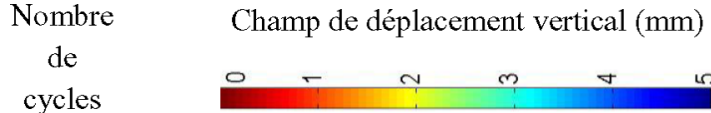

1

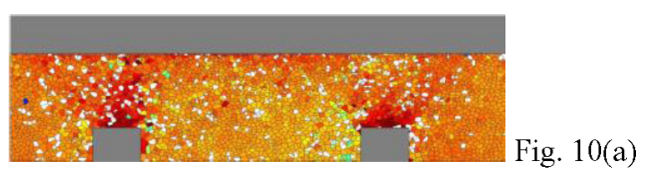

2

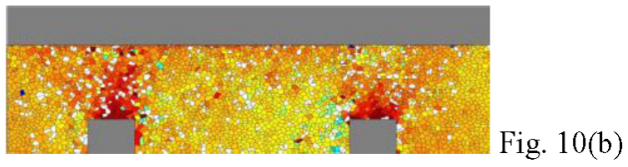

3

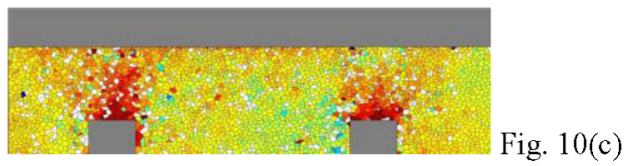

4

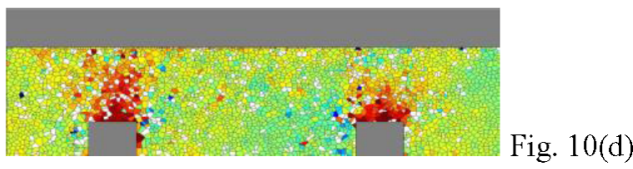

5

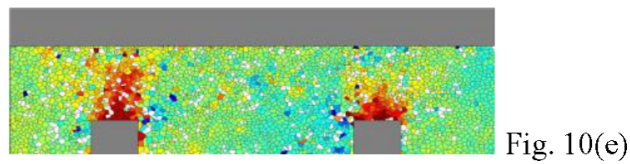

6

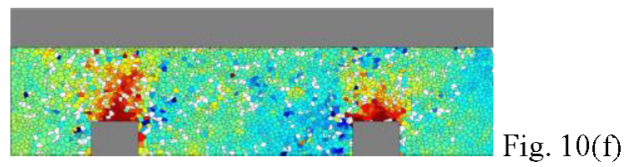

Fig. 10. Champ de déplacement vertical à la fin de chacun de six premiers cycles pour l'essai C1_D.

Fig. 10. Vertical displacement field at the end of the six first cycles for the test $C 1 \_D$.

$20 \mathrm{kPa}$ que la majeure partie du tassement se développe. Cette analyse confirme les résultats obtenus par le capteur CD3 dans la zone centrale du modèle (Fig. 7).

\section{4 Étude paramétrique}

\section{1 Étude de l'influence de préchargement du système avec dallage}

Les résultats de l'essai C1_D sont ici comparés avec ceux de l'essai C2_D afin de déterminer l'influence d'un préchargement de l'ouvrage avant l'application des cycles sur le 
Champ de déplacement vertical ( $\mathrm{mm})$

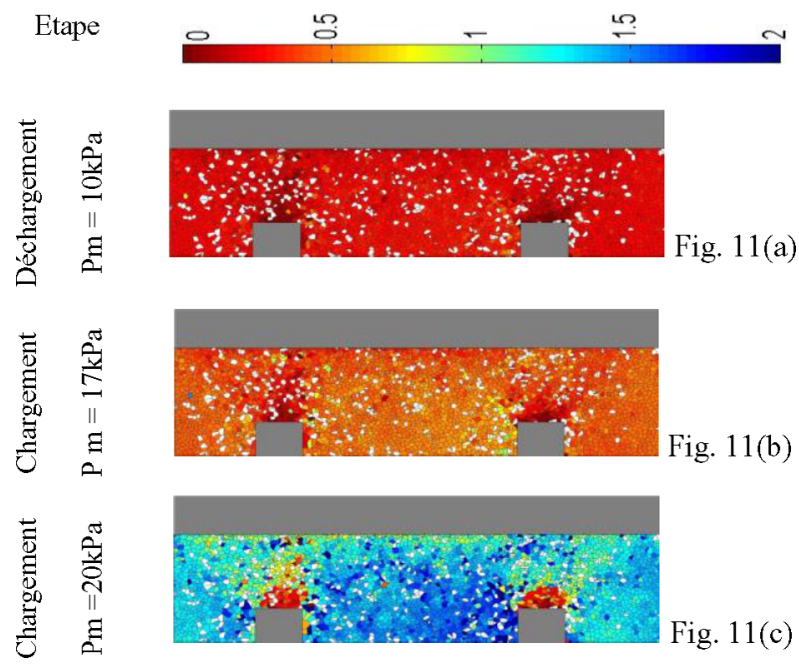

Fig. 11. Évolution du champ de déplacement vertical durant le premier cycle de chargement-déchargement pour l'essai C1_D.

Fig. 11. Evolution of the vertical displacement field during the first cycle of loading-unloading for the test $C 1 \_D$. comportement ultérieur du système. Les deux essais sont réalisés avec la même configuration géométrique et avec les mêmes valeurs de contraintes minimales et maximales du chargement cyclique. Le préchargement du système à $30 \mathrm{kPa}$ pour l'essai C2 D mène à une "surconsolidation » du sol support (notons cependant qu'avec le sol analogue utilisé il n'y a pas à proprement parler de « consolidation », observée pour les vrais sols, au sens de la théorie de Terzaghi).

La valeur de la force moyenne en tête des quatre inclusions centrales est sensiblement la même pour les deux essais cycliques C1_D et C2_D (Fig. 12), ce qui signifie que le préchargement du système, par l'application d'une pression à la surface $P m=30 \mathrm{kPa}$ avant l'application du chargement cyclique, ne change pas l'influence des cycles en termes de report de charge vers les têtes des inclusions rigides. L'efficacité moyenne du système, définie suivant Hewlett et Randolph (1988), comme étant le rapport entre la force transférée vers la tête de l'inclusion rigide et la force totale appliquée sur la zone d'influence de cette dernière, n'est pas influencée par ce changement de l'état de « consolidation » du sol compressible (Fig. 13).

L'évolution du tassement D3 avec la pression Pm appliquée en surface pour les deux essais C1 D et C2 D montre une bonne répétabilité des tassements entre les deux essais et pour les deux paliers de chargement monotone (Fig. 14). Le préchargement du

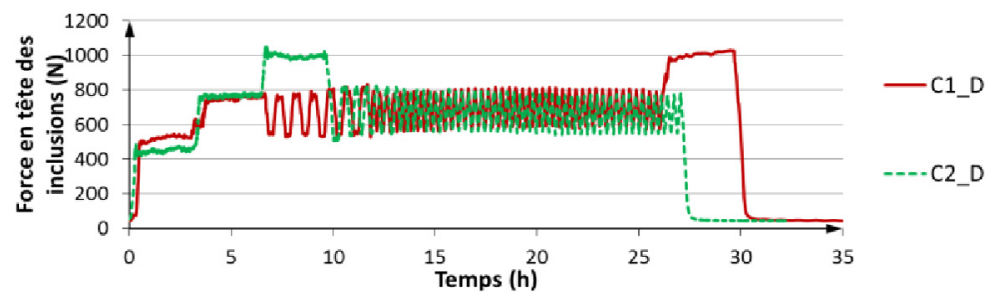

Fig. 12. Évolution de la force moyenne en tête des quatre inclusions centrales avec le temps pour les essais cycliques avec et sans préchargement (C1_D et C2_D).

Fig. 12. Evolution of the average force at the head of four central inclusions for the tests $C 1 \_D$ and $C 2 \_D$.

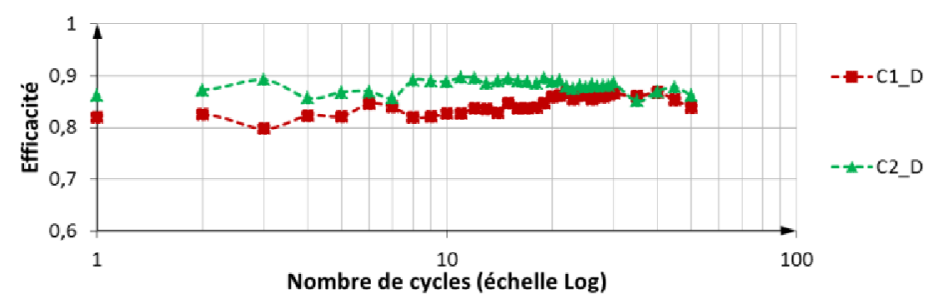

Fig. 13. Évolution de l'efficacité moyenne des quatre inclusions centrales au pic des cycles en fonction du nombre de cycles pour les essais C1_D et $\mathrm{C} 2$ D.

Fig. 13. Evolution of the average efficiency at the head of four central inclusions at the peak of the cycles versus the number of cycles for the tests C1_D and $C 2 \_D$.

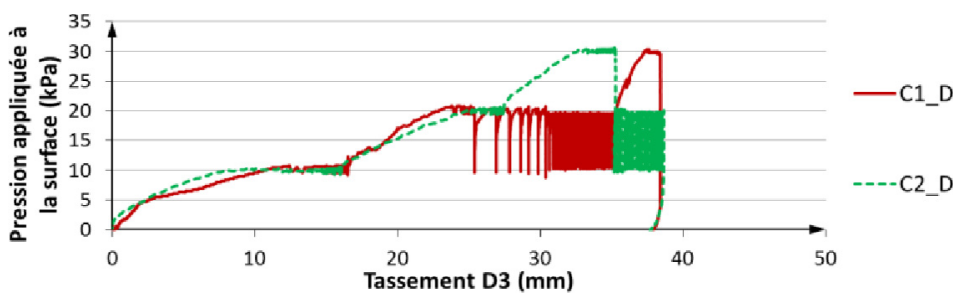

Fig. 14. Évolution du tassement $\mathrm{D} 3$ avec la pression appliquée à la surface pour les essais C1_D et C2_D.

Fig. 14. Settlement evolution of $D 3$ with the applied pressure at the surface for the tests $C 1 \_D$ and $C 2 \_D$. 


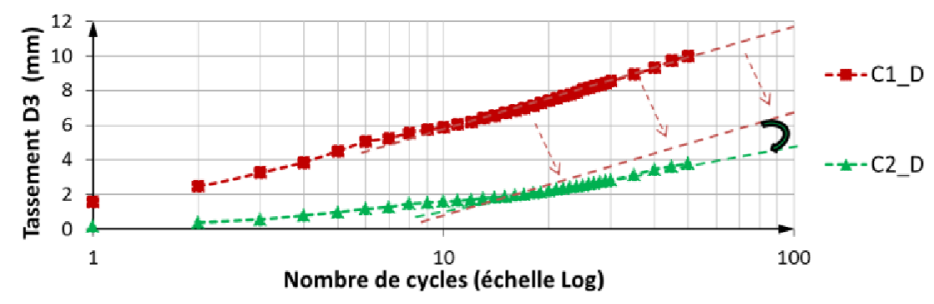

Fig. 15. Évolution de l'accumulation de tassement au pic des cycles en fonction du nombre de cycles pour les essais C1_D et C2_D.

Fig. 15. Settlement accumulation at the peak of the cycles versus of the number of cycles for the tests $C 1$ D and $C 2 \_D$.

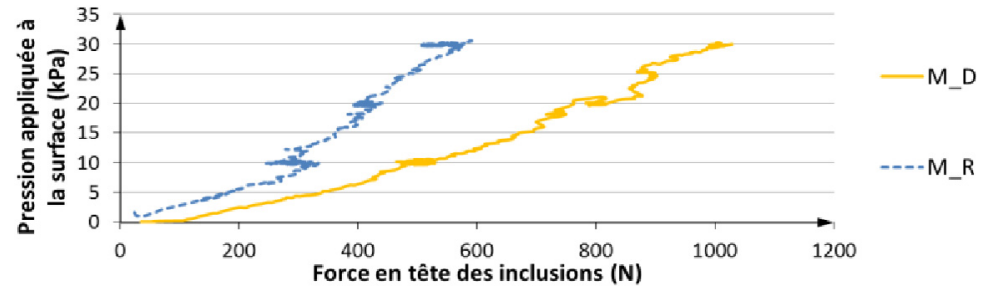

Fig. 16. Évolution de la force moyenne en tête des quatre inclusions centrales avec la pression appliquée en surface pour les essais monotones avec et sans dalle (M_D et M_R).

Fig. 16. Evolution of the average force at the head of four central inclusions for the tests $M_{-} D$ and $M_{-} R$.

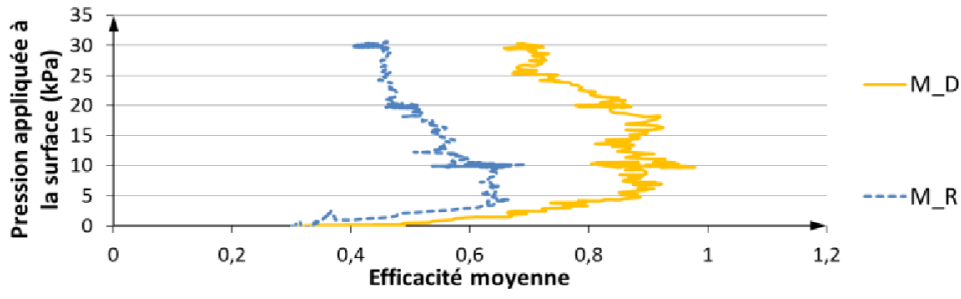

Fig. 17. Évolution de l'efficacité moyenne en tête des inclusions avec la pression appliquée en surface pour les essais monotones avec et sans dalle (M_D et M_R).

Fig. 17. Evolution of the average efficiency at the head of four central inclusions for the tests $M_{-} D$ and $M_{-} R$.

système dans l'essai C2_D n'empêche cependant pas l'accumulation des tassements durant les cycles, sa valeur étant égale à $4 \mathrm{~mm}$. En revanche, cette accumulation vaut seulement $40 \%$ de celle mesurée dans l'essai C1_D (10 mm). Enfin, on peut noter que le tassement final est quasiment identique pour les deux essais. Cela signifie que le fait de précharger le système, et donc précharger le sol support avant d'appliquer la sollicitation cyclique, ne change pas la valeur du tassement final du sol mais amène à une réduction importante de l'accumulation de tassement durant le chargement cyclique.

La Figure 15, qui représente en échelle logarithmique l'accumulation des tassements durant les cycles, montre que, pour l'essai C2_D, environ $40 \%$ de l'accumulation totale de tassement durant le chargement cyclique se fait pendant les dix premiers cycles. Cette proportion avait été trouvée égale à $60 \%$ pour l'essai C1_D (Fig. 8). Le préchargement du système introduit également une diminution de la pente de la droite qui définit l'allure linéaire des deux courbes. Cela signifie que la réduction de l'accumulation de tassement serait conservée lors de l'application d'un plus grand nombre de cycles.

\section{2 Étude de l'influence du dallage}

La réalisation des essais en appliquant le chargement directement à la surface de la PTC permet d'étudier l'influence de la présence ou non d'une dalle rigide à la surface de la PTC sur le comportement du système. Cette influence est d'abord étudiée sous chargement monotone en comparant les résultats de l'essai M_D avec ceux de l'essai M_R réalisé sans dalle rigide à la surface.

La force moyenne en tête des inclusions apparaît près de deux fois plus faible pour l'essai M_R que pour l'essai M_DR (Fig. 16). Cela montre que la présence de la dalle rigide favōrise largement, sous chargement monotone, le report de charge vers la tête des inclusions, ce qui était clairement attendu.

Si l'on suit les évolutions de l'efficacité avec le chargement monotone (Fig. 17), on observe la même tendance pour les deux conditions aux limites (augmentation rapide puis diminution jusqu'à une valeur quasi stabilisée) mais l'efficacité pour l'essai M_R reste toujours $20 \%$ plus faible que celle de l'essai M D.

On observe de manière logique que les tassements sont plus importants en absence de la dalle pour l'essai M_R (Fig. 18). La diminution du transfert de charge vers la tête des inclusions rigides observée pour l'essai $\mathrm{M} \_\mathrm{R}$ mène en effet à une augmentation de la pression appliquée sur le sol compressible et par la suite à plus de tassement de ce dernier.

L'influence sous chargement cyclique de la présence de la dalle rigide à la surface de la PTC est étudiée en comparant, d'une part, les essais C1_D et C1_R et, d'autre part, les essais C2_D et C2_R (ce qui permet en plus d'identifier l'effet combiné du préchargement du système avant l'application 


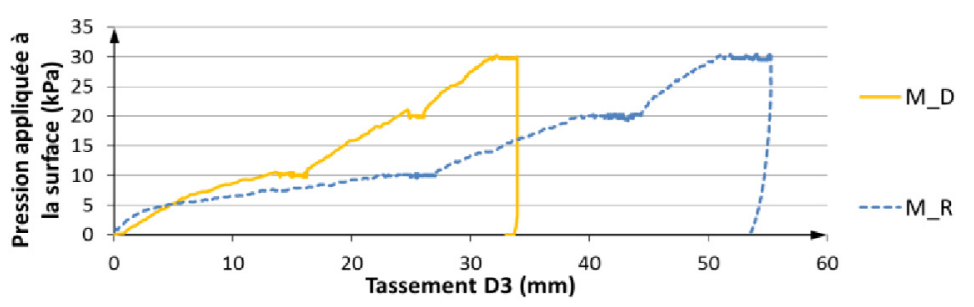

Fig. 18. Évolution du tassement D3 avec la pression appliquée en surface pour les essais monotones avec et sans dalle (M_D et M_R).

Fig. 18. Settlement evolution of D3 with the applied pressure at the surface for the tests $M \_D$ and $M_{-} R$.

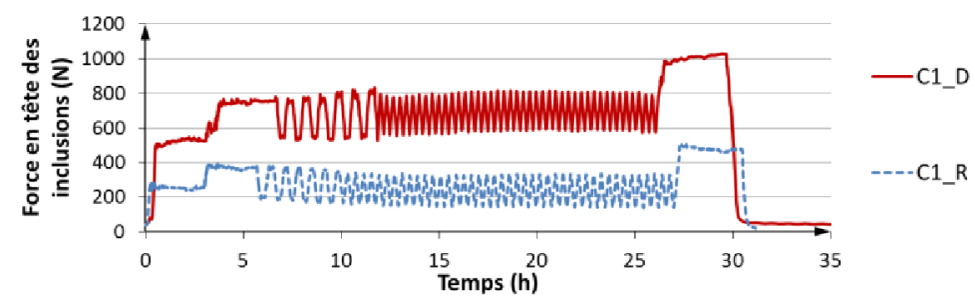

Fig. 19. Évolution de la force moyenne en tête des quatre inclusions centrales avec le temps pour les essais cycliques 1 avec et sans dalle (C1_D et C1_R).

Fig. 19. Evolution of the average force at the head of four central inclusions for the tests $C 1 \_D$ and $C 1_{-} R$.

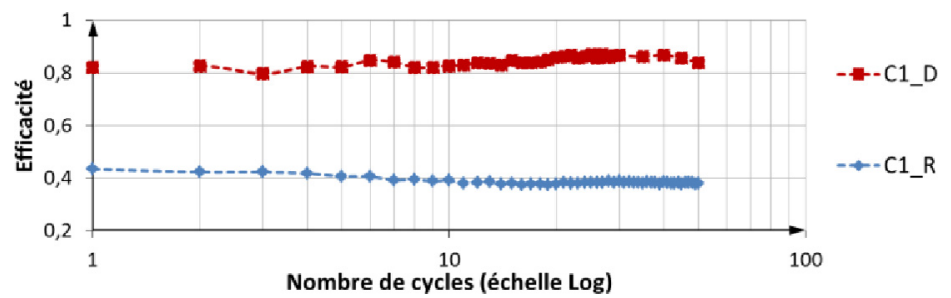

Fig. 20. Efficacité moyenne en tête des inclusions en fonction du nombre de cycles pour les essais cycliques 1 avec et sans dalle (C1_D et C1_R). Fig. 20. Evolution of the average efficiency at the head of four central inclusions at the peak of the cycles versus the number of cycles for the tests $C 1 \_D$ and $C 1 \_R$.

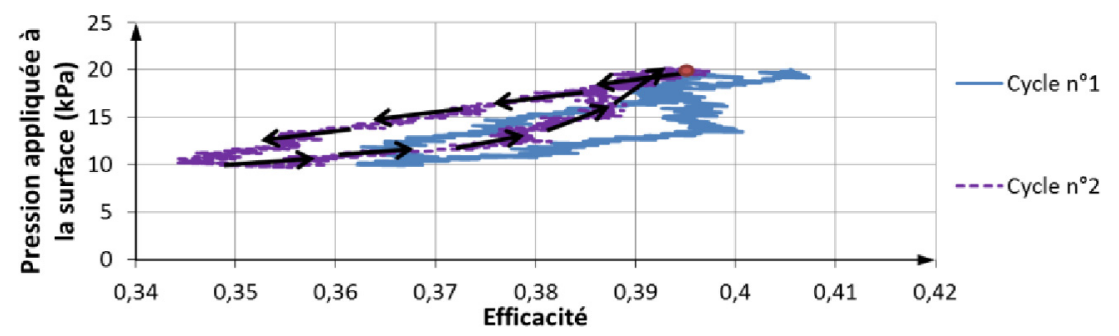

Fig. 21. Évolution de l'efficacité moyenne en tête des inclusions sur la phase de chargement-déchargement du premier et deuxième cycles pour l'essai cyclique sans dallage $\mathrm{C} 2$ R.

Fig. 21. Evolution of the average efficiency during the first two cycles of loading-unloading for the test C2_R.

des cycles). On observe sur la Figure 19 que l'absence de la dalle rigide dans l'essai $\mathrm{C} 1$ _R mène à une légère diminution de la force moyenne en tête des inclusions au pic des dix premiers cycles, suivie d'une stabilisation, ce qui se traduit par une diminution de l'efficacité moyenne du système (Fig. 20). La courbe de variation de l'efficacité est plus chahutée pour l'essai C1_D que pour l'essai C1_R réalisé sans dalle, ce qui peut être lié à la concentration de charge sur les têtes d'inclusions et à l'influence de l'aspect granulométrie de la PTC qui sont plus importants en présence de la dalle.
Les mêmes observations en termes d'évolution des tassements et de l'efficacité ont pu être faites dans le cas du chargement cyclique $\mathrm{C} 2$ appliqué sur un système préchargé à $30 \mathrm{kPa}$ avant l'application des cycles. Cela signifie que, en absence de dallage, le préchargement du système n'influence pas l'évolution du transfert de charge observée durant le chargement cyclique.

Une analyse fine de l'évolution de l'efficacité durant les deux premiers cycles de l'essai C2_R (Fig. 21) montre que l'efficacité diminue légèrement en passant du premier cycle au deuxième et que l'efficacité diminue durant le déchargement et 


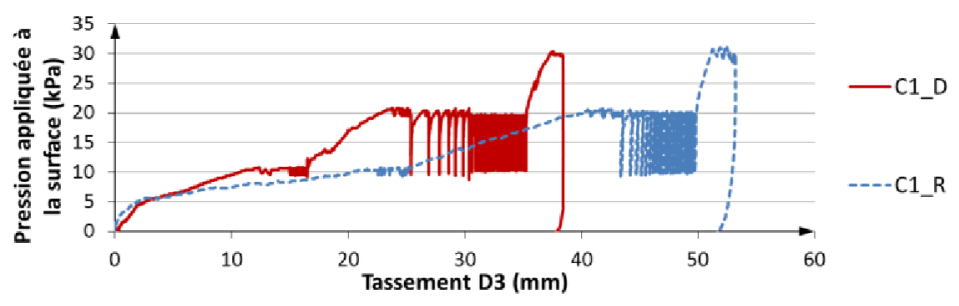

Fig. 22. Évolution du tassement $\mathrm{D} 3$ avec la pression appliquée en surface pour les essais C1_D et C1_R. Fig. 22. Settlement evolution of $D 3$ with the applied pressure at the surface for the tests $C 1{ }_{-} D$ and $C \overline{1} \_$.

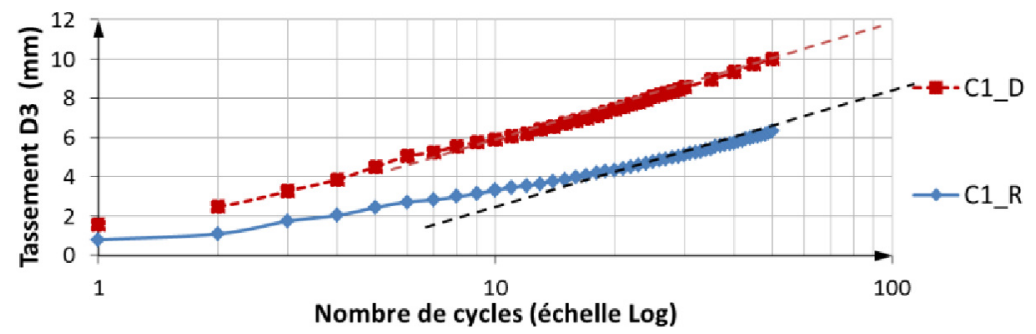

Fig. 23. Évolution de l'accumulation de tassement au pic des cycles en fonction du nombre de cycles pour les essais C1_D et C1_R.

Fig. 23. Settlement evolution of $D 3$ with the applied pressure at the surface for the tests $C 1 \_D$ and $C 1 \_R$.

ré-augmente lors du rechargement. Cette diminution progressive d'efficacité est liée au comportement irréversible du matelas granulaire et met en évidence la plasticité locale du matelas au-dessus des inclusions quand la pression appliquée à la surface atteint $20 \mathrm{kPa}$.

L'accumulation de tassement mesurée durant le chargement cyclique pour l'essai sans dallage $\mathrm{C} 1 \_\mathrm{R}$ est inférieure de $50 \%$ à celle mesurée en présence de la dalle à la surface $(10 \mathrm{~mm}$ avec dalle et seulement $5 \mathrm{~mm}$ sans dalle) (Fig. 22). Ce résultat provient du fait que, dans l'essai réalisé sans dalle rigide, le sol a subi des tassements déjà importants avant l'application des cycles, qui empêchent une grande accumulation ultérieure de tassement durant l'application des cycles. Sans surprise, on observe que le cas sans dalle est nettement défavorable en matière de tassement total sous chargement monotone et cyclique.

La pente de la droite qui définit l'évolution en régime permanent du tassement durant le chargement cyclique en fonction du nombre de cycles en échelle logarithmique est sensiblement la même pour les deux essais C1_D et C1_R (Fig. 23). Cela signifie que même si les phénomènes de départ sont légèrement différents, les mécanismes mis en jeu dans l'évolution avec les cycles sont semblables quelles que soient les conditions aux limites appliquées à la surface de la PTC.

Sous le chargement cyclique $\mathrm{C} 2$ appliqué sur un système préchargé, l'accumulation de tassement est plus importante en présence de la dalle de manière analogue au cas du chargement cyclique $\mathrm{C} 1$ (Fig. 22).

\section{Modèle numérique et résultats}

Les résultats expérimentaux obtenus aux chapitres précédents vont être utilisés ici comme référence pour le développement et la validation d'un modèle numérique en milieu continu. La modélisation numérique est effectuée avec le logiciel FLAC ${ }^{3 \mathrm{D}}$ (version 4.00.32, Itasca, 2009). Profitant des conditions de symétrie dans le modèle physique, la

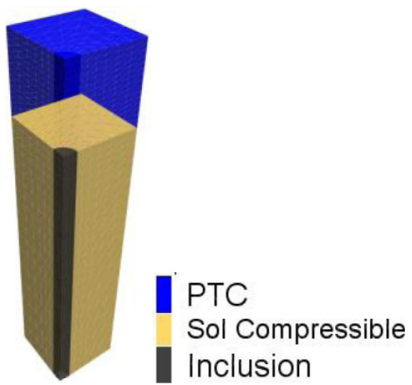

Fig. 24. Vue schématique du modèle numérique en 3D.

Fig. 24. 3D schematic view of the numerical model.

Tableau 4. Caractéristiques mécaniques (élastique linéaire) de l'inclusion rigide.

Table 4. Mechanical characteristics (linear elastic) of the rigid inclusion.

\begin{tabular}{llc}
\hline$\Upsilon_{\mathrm{d}}\left(\mathrm{kN} / \mathrm{m}^{3}\right)$ & $v$ & $E(\mathrm{MPa})$ \\
\hline 27 & 0,3 & 72500 \\
\hline
\end{tabular}

modélisation numérique nous permet de ne considérer qu'un seizième de la zone centrale (Fig. 24). Pour satisfaire aux conditions de symétrie, les déplacements au niveau des axes de symétrie sont bloqués horizontalement. La limite inférieure est bloquée verticalement. Des interfaces ont été mises en place sur les bords de l'inclusion et entre le sol compressible et la PTC, ce qui permet un déplacement relatif sol compressible/ inclusion et $\mathrm{PTC} / \mathrm{sol}$ compressible, et donc de modéliser l'effet d'accrochage entre le sol et l'inclusion.

L'inclusion en aluminium, dont la rigidité est très importante par rapport à celle du matelas de transfert des charges et celle du sol compressible, est modélisée avec une loi de comportement élastique linéaire (Tab. 4). 
Tableau 5. Paramètres du modèle Cam-Clay modifié utilisés pour modéliser le sol compressible.

Table 5. Parameters used to model the compressible soil (modified Cam-Clay).

\begin{tabular}{lllllll}
\hline$\lambda$ & $\kappa$ & $M$ & $G(\mathrm{MPa})$ & $P_{1}(\mathrm{kPa})$ & $v_{\lambda}$ & $P c_{0}(\mathrm{kPa})$ \\
\hline 0,8 & 0,01 & 1,3 & 10 & 1 & 10 \\
\hline
\end{tabular}
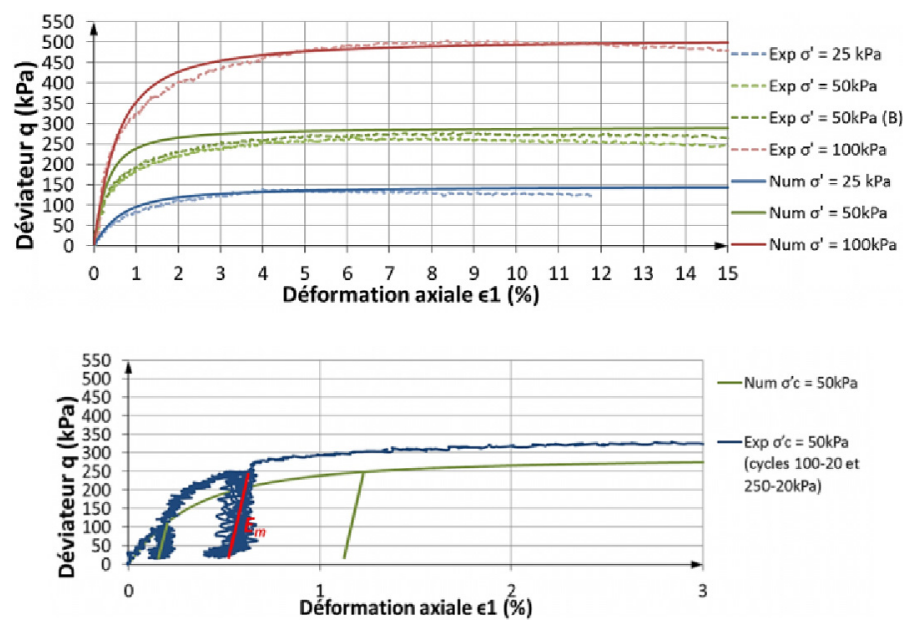

Fig. 25. Confrontation des résultats des essais triaxiaux donnés par la loi CY-soil avec les résultats expérimentaux (q versus $\in 1$ ).

Fig. 25. Confrontation of the triaxial test results given by the CY-soil model with experimental results ( $q$ versus $\in 1)$.

Tableau 6. Paramètres utilisés pour modéliser la plateforme de transfert de charge granulaire (modèle CY-soil).

Table 6. Parameters used to model the granular load transfer platform (of CY-soil).

\begin{tabular}{lllllllll}
\hline$G_{r e f}(\mathrm{MPa})$ & $\Phi_{\mathrm{f}}\left({ }^{\circ}\right)$ & $c(\mathrm{kPa})$ & $\Psi_{\mathrm{f}}\left(^{\circ}\right)$ & $v$ & $P_{r e f}(\mathrm{kPa})$ & $R_{f}$ & $\beta$ & $\alpha$ \\
\hline 400 & 45 & 0 & 10 & 0,4 & 50 & 0,95 & 0,35 & 1 \\
\hline
\end{tabular}

Le modèle Cam-Clay modifié a été utilisé pour la modélisation du comportement du sol compressible. Des modèles adaptés aux comportements des sols compressibles sous chargement cyclique existent dans la littérature (Al-Tabbaa, 1987 ; Al-Tabbaa et Wood, 1989, etc.), mais ne seront pas mis en œuvre dans cet article. Cela nous permet d'éliminer l'influence du chargement cyclique sur le comportement du sol compressible lors des simulations numériques et de ne se focaliser alors que sur l'évolution des phénomènes au sein de la PTC. Le jeu de paramètres permettant de s'approcher des résultats issus des essais œdométriques est donné dans le Tableau 5.

Le comportement du sol composant le matelas est simulé par le modèle élasto-plastique «CY-soil » (pour « Cap Yield soil model », Itasca, 2009) implanté dans le code FLAC ${ }^{3 \mathrm{D}}$, afin d'étudier sa capacité à représenter le comportement du sol sous chargement cyclique. Nous cherchons donc à valider l'utilisation de cette loi de comportement à partir des résultats des essais expérimentaux. Le modèle CY-soil est caractérisé par une surface de charge en cisaillement analogue à celle de MohrCoulomb et une surface de fermeture (appelée « cap ») se refermant sur l'axe des compressions isotropes, de façon à générer des déformations plastiques lors d'une compression isotrope. En outre, le modèle CY-soil permet de représenter la variation du module élastique en fonction de la déformation volumique plastique. Il rend également compte d'une différence entre les modules de chargement et de déchargement-rechargement. Les paramètres du modèle sont identifiés à partir d'essais expérimentaux triaxiaux monotones et cycliques. L'étude de sensibilité des paramètres du modèle CY-soil nous a permis d'ajuster le jeu de paramètres permettant d'approcher les résultats expérimentaux pour un chargement monotone (Fig. 25). Le jeu de paramètres résultant de cette calibration est donné dans le Tableau 6.

Pour le cas sans dallage et sous chargement monotone, les résultats numériques correspondent bien aux résultats expérimentaux en termes d'efficacité et de tassement respectivement (Fig. 26 et 27). La Figure 26 montre notamment qu'au-delà d'une pression $P m$ égale à $10 \mathrm{kPa}$ l'efficacité numérique se stabilise autour d'une valeur égale à $50 \%$.

La modélisation numérique sous chargement cyclique sans dallage permet de représenter la diminution de l'efficacité observée expérimentalement durant le chargement cyclique et qui se stabilise au bout de dix cycles (Fig. 28). Cela indique que le transfert de charge vers la tête de l'inclusion est gardé constant après les dix premiers cycles.

L'évolution de la distribution des contraintes dans la PTC au cours des cycles à une hauteur $z=0,025 \mathrm{~m}$ au-dessus de la base de la PTC représentée sur la Figure 29 montre clairement 


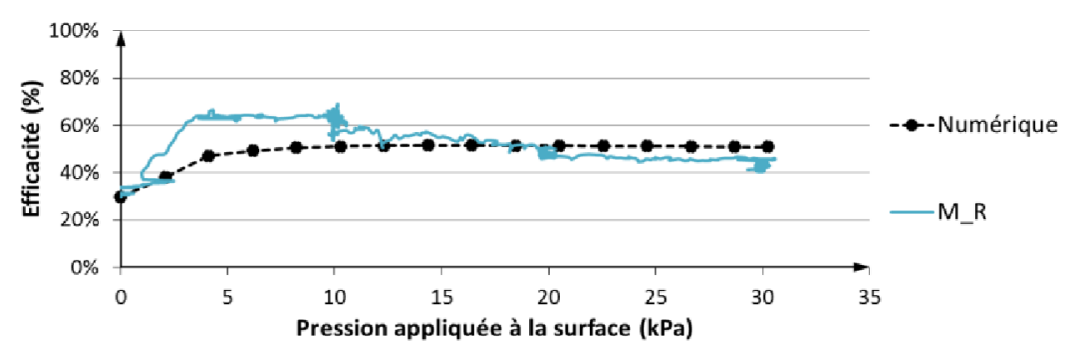

Fig. 26. Évolution expérimentale et numérique de l'efficacité moyenne en tête des inclusions avec la pression appliquée à la surface pour l'essai monotone sans dallage M_R.

Fig. 26. Experimental and numerical evolution of the average efficiency with the pressure applied at the surface for the test $M \_R$.

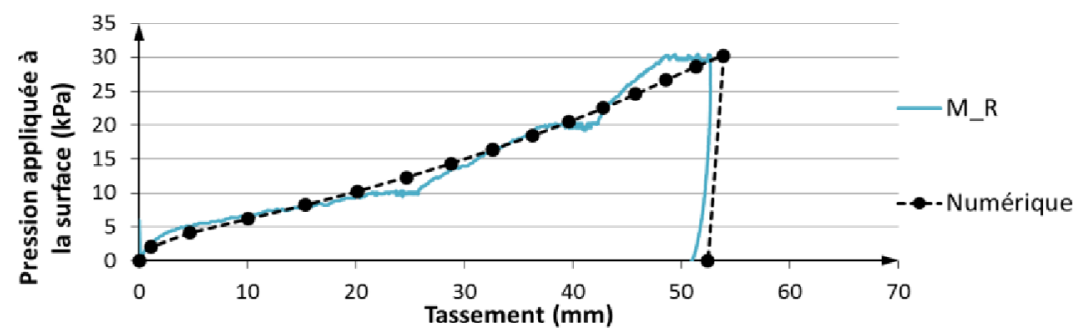

Fig. 27. Évolution expérimentale et numérique du tassement D3 avec la pression appliquée en surface pour l'essai M_R.

Fig. 27. Experimental and numerical evolution of the settlement D3 with the pressure applied at the surface for the test $M_{-} R$.

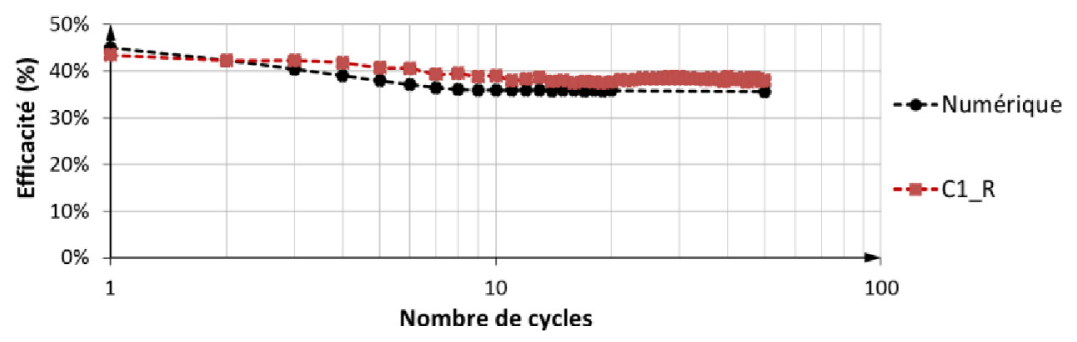

Fig. 28. Efficacité expérimentale et numérique en fonction du nombre de cycles pour l'essai cyclique sans dallage C1_R.

Fig. 28. Experimental and numerical evolution of the average efficiency at the peak of the cycles versus the number of cycles for the test $C 1$ R.

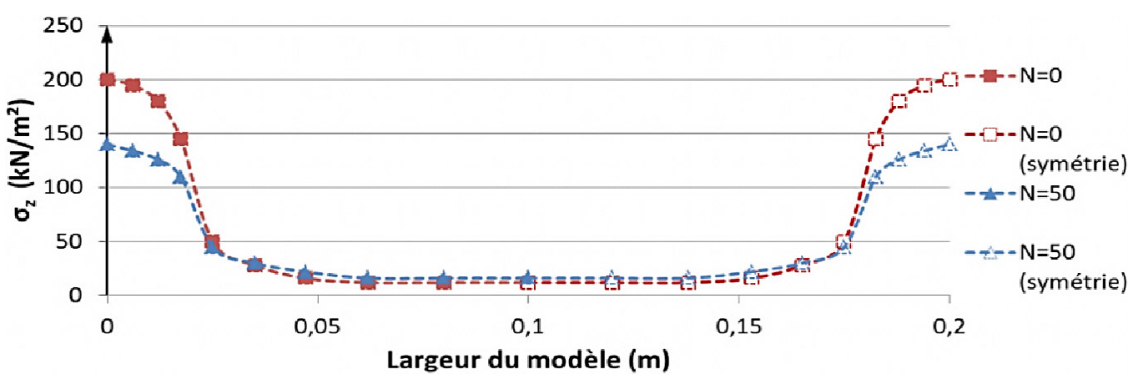

Fig. 29. Contraintes verticales dans la PTC à une hauteur $z=0,025 \mathrm{~m}$ au-dessus de la base de la PTC en fonction de la largeur de modèle pour l'essai C1_R.

Fig. 29. Vertical stresses in the LTP at a height $z=0.025 \mathrm{~m}$ above the base of the LTP versus the distance from the inclusion center for the test C1_R.

le mécanisme d'effet de voûte avant l'application du chargement cyclique (pour $n=0$ ), où les contraintes sont bien plus importantes à l'aplomb de l'inclusion (distance à l'axe de l'inclusion $<0,0175 \mathrm{~m}$ ) qu'au centre du modèle. La répartition des contraintes à cette profondeur de la PTC commence à avoir une allure uniforme à partir d'une distance horizontale à l'axe de l'inclusion supérieure à $0,05 \mathrm{~m}$. Après $n=50$ cycles, on observe que les contraintes à l'aplomb de l'inclusion subissent une diminution forte (de 200 à $140 \mathrm{kPa}$ ) accompagnée d'une augmentation des contraintes à l'aplomb du sol compressible. Cela signifie que le report de charge par effet de voûte, développé sous chargement monotone, est partiellement « détruit » au cours des cycles.

Une accumulation de tassement est obtenue numériquement au cours du chargement cyclique (Fig. 30), d'une amplitude sensiblement voisine de ce qui est mesuré 


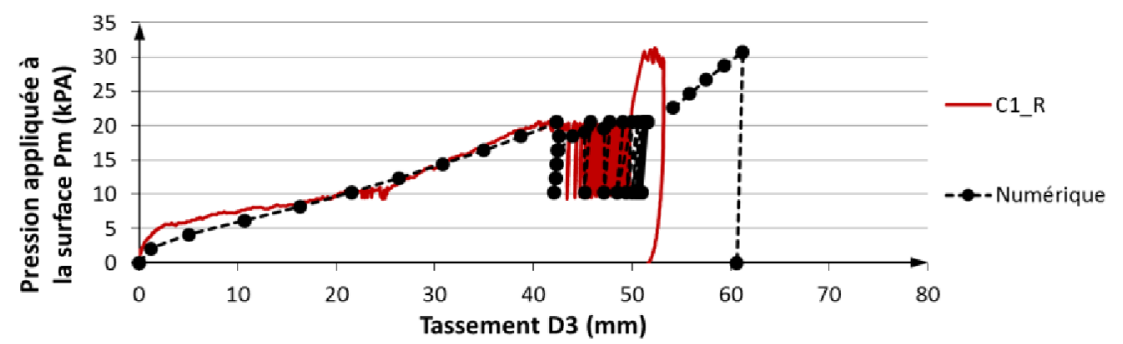

Fig. 30. Évolution expérimentale et numérique du tassement $\mathrm{D} 3$ avec la pression appliquée en surface pour l'essai $\mathrm{C} 1 \mathrm{R}$.

Fig. 30. Experimental and numerical evolution of the settlement D3 with the pressure applied at the surface for the test C1_R.

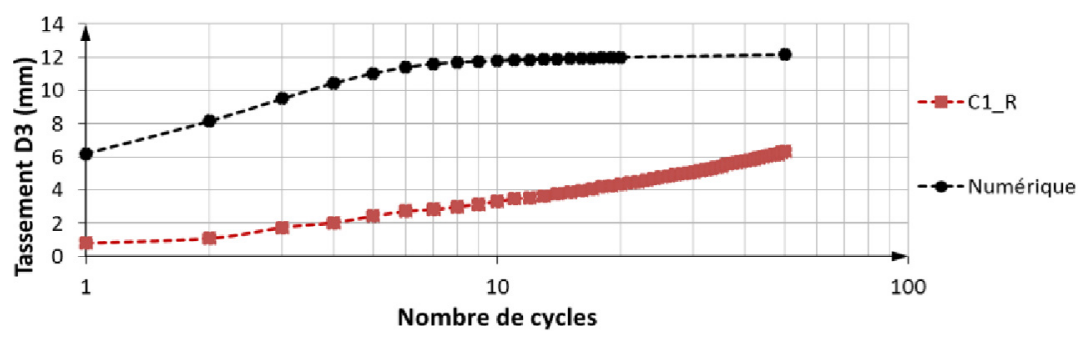

Fig. 31. Évolution de l'accumulation de tassement numérique et expérimentale au pic des cycles en fonction du nombre de cycles pour l'essai C1_R.

Fig. 31. Experimental and numerical evolution of the settlement D3 versus the number of cycles for the test $C 1 \_R$.

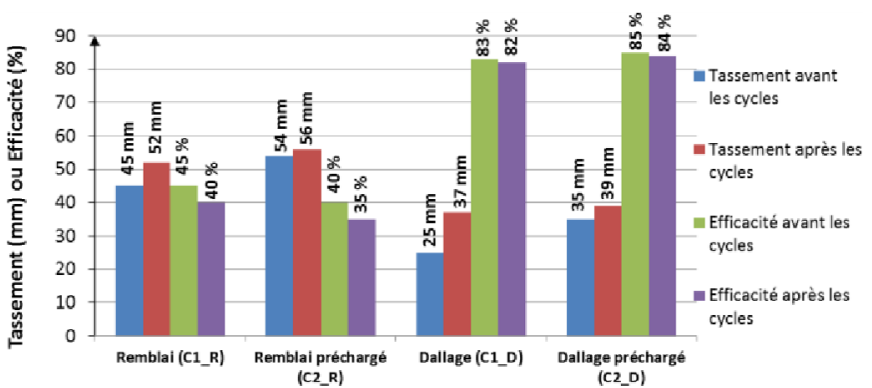

Fig. 32. Résultats expérimentaux avant et après l'application des cycles.

Fig. 32. Experimental results before and after the application of cycles.

expérimentalement. En revanche, contrairement au cas expérimental, le chargement cyclique n'influence pas la pente de la courbe de tassement lors d'un chargement monotone ultérieur.

La Figure 31 montre que la modélisation numérique surestime toutefois l'accumulation de tassement au cours des dix premiers cycles (d'environ trois fois), cette différence est due notamment au premier cycle. Au-delà de dix cycles appliqués, le tassement du sol compressible se stabilise, cette observation est cohérente avec la Figure 28 qui montre que le transfert de charge vers la tête de l'inclusion obtenu numériquement se stabilise au bout de dix cycles et que par suite la contrainte appliquée sur le sol compressible ne varie pas.

\section{Conclusion}

Une campagne d'essais sur un modèle réduit tridimensionnel développé au laboratoire 3SR a été réalisée dans le but d'étudier le comportement sous chargement cyclique de la technique de renforcement de sol compressible par inclusions rigides. Cette étude expérimentale a permis également d'étudier l'influence du préchargement du système avant l'application des cycles et l'influence de la présence d'un dallage sur le comportement du système.

Une accumulation de tassement a été observée durant le chargement cyclique pour les deux cas : sans et avec dallage. Il s'agit d'une accumulation de tassement qui se fait avec un rythme décroissant sans arriver à une stabilisation à la fin de la sollicitation cyclique.

Il a été montré également que la présence de la dalle augmente le transfert de charge et diminue le tassement du sol compressible sous chargement monotone. Cependant, la présence de la dalle mène à une accumulation de tassement plus importante au cours des cycles. Néanmoins, le tassement total reste plus faible en présence de la dalle.

Les mesures montrent que le préchargement du système, que ce soit sans ou avec dallage, aide à diminuer l'accumulation de tassement observée durant le chargement cyclique sans pouvoir toutefois l'empêcher. La Figure 32 synthétise l'ensemble des résultats expérimentaux obtenus avant et après l'application des cycles, en matière d'efficacité ou de tassement.

Une modélisation numérique tridimensionnelle en milieu continu, utilisant le logiciel FLAC ${ }^{3 \mathrm{D}} \mathrm{V} 4.00 .32$, a été mise en œuvre afin d'améliorer la compréhension du mode de fonctionnement de ce type d'ouvrage sous chargement cyclique. La modélisation numérique effectuée a permis de reproduire qualitativement le comportement de ce type d'ouvrage et de retrouver les observations faites sur le modèle réduit sous chargement monotone et cyclique. De manière plus précise (et quantitative), les confrontations entre résultats numériques et expérimentaux sont encourageantes mais font cependant apparaître que la réduction de l'efficacité et l'accumulation de tassement relevées durant le chargement cyclique sur les essais expérimentaux sans dallage sont 
surestimées par la modélisation numérique. La mise en œuvre d'un modèle de comportement plus adapté à la prise en compte effective de sollicitations cycliques est donc requise. Il serait également nécessaire d'adopter une loi de comportement cyclique pour représenter le comportement du sol compressible.

Le modèle physique permettra, grâce à sa modularité, la réalisation d'essais avec différents matériaux et hauteurs de la PTC afin de déterminer l'influence de cette dernière en termes d'épaisseur, d'angle de frottement, de granularité et de cohésion (dans le cas des sols traités), etc. Il est également possible de réaliser l'étude d'autres configurations de chargement cyclique afin de déterminer l'influence de l'amplitude et du niveau de chargement cyclique sur le comportement du système.

Remerciements. Les auteurs remercient la direction technique et de la recherche de la Fédération nationale des travaux publics (FNTP) pour l'aide financière apportée à cette étude. Les auteurs tiennent à exprimer leur gratitude à J. Canou (ENPC), L. Thorel (IFSTTAR), P. Gotteland (FNTP), S. Brûlé (Menard) ainsi qu'à L. Briançon (INSA Lyon) pour leurs conseils sur ce travail. De nombreux remerciements vont également à $\mathrm{G}$. Combe (3SR laboratoire) pour son aide dans la pratique du programme « Tracker», ainsi que G. Vian et J. B. Toni pour le soutien technique.

\section{Références}

Al-Tabbaa A. 1987. Permeability and stress-strain response of speswhite kaolin. Thèse de doctorat, université de Cambridge.

Al-Tabbaa A, Wood DM. 1989. An experimentally based bubble model for clay. In: Proc. 3rd Int. Conf. on Numerical Models in Geomechanics, Niagara Falls, pp. 91-99.

Antoine PC. 2010. Étude des dalles sur sols renforcés au moyen d'inclusions rigides ou non. Thèse de doctorat, faculté des sciences appliquées (Bruxelles).

Arwanitaki A, Triantafyllidis T. 2006. Mehrlagig mit Geogittern bewehrte Erdkörper über pfahlartigen Gründungselementen. Numerische Simulation des Verformungsverhaltens unter statischer und zyklischer Einwirkung. Bautechnik 83: 695-707, en allemand.

Baudouin G. 2010. Sols renforcés par inclusions rigides : modélisation physique en centrifugeuse de remblais et de dallage. Thèse de doctorat, université de LUNAM.

Briançon L, Dias D, Simon S. 2015. Monitoring and numerical investigation of a rigid inclusions-reinforced industrial building. Can. Geotech. J. 52: 1-13, doi: 10.1139/cgj-2014-0262.

Chen YM, Cao WP, Chen RP. 2008. An experimental investigation of soil arching within basal reinforced and unreinforced piled embankments. Geotext. Geomembr. 26: 164-174.

Chevalier B, Villard P, Combe G. 2011. Investigation of load-transfer mechanisms in geotechnical earth structures with thin fill platforms reinforced by rigid inclusions. Int. J. Geomech. 11: 239-250.

Combe G, Richefeu V. 2013. Tracker: a Particle Image Tracking (PIT) technique dedicated to nonsmooth motions involved in granular packings. In: Powders and Grains 2013, July 8-12, UNSW, Sydney, Australia.

De Pasquale A. 2011. Cyclic behaviour of geotechnical structures involving granular mats. Mémoire du Master International, université J.-Fourier (Grenoble), 41 p.

Demerdash MA. 1996. An experimental study of piled embankments incorporating geosynthetic basal reinforcement. Thèse de doctorat, University of Newcastle-upon-Tyne.
Dinh AQ. 2010. Étude sur modèle physique des mécanismes de transfert de charge dans les sols renforcés par inclusions rigides. Application au dimensionnement. Thèse de doctorat, École des Ponts (Paris).

Ellis EA, Aslam R. 2009a. Arching in piled embankments: comparison of centrifuge tests and predictive methods. Part 1 of 2. Ground Eng. 42: 34-38.

Ellis EA, Aslam R. 2009b. Arching in piled embankments: comparison of centrifuge tests and predictive methods. Part 2 of 2. Ground Eng. 42: 28-31.

Eskisar T, Otani J, Hironaka J. 2012. Visualization of soil arching on reinforced embankment with rigid pile foundation using X-ray CT. Geotext. Geomembr. 32: 44-54.

Han J, Gabr MA. 2002. Numerical analysis of geosynthetic-reinforced and pile-supported earth platforms over soft soil. J. Geotech. Geoenviron. Eng. 128: 44-53.

Han J, Bhandari A. 2009. Evaluation of geogrid-reinforced pilesupported embankments under cyclic loading using discrete element method. In: ASCE Conf. Advances in Ground Improvement (GSP 188), Proc. of the 2009 US - China Workshop on Ground Improvement Technologies, $10 \mathrm{p}$.

Han J, Bhandari A, Wang F. 2011. DEM analysis of stresses and deformations of geogrid-reinforced embankments over piles. Int. J. Geomech. 12: 340-350.

Heitz C. 2006. Bodengewölbe unter ruhender und nichtruhender Belastung bei Berücksichtigung von Bewehrungseinlagen aus Geogittern. Schriftenreihe Geotechnik, Universität Kassel. Heft 19. Kassel: Kassel University Press GmbH, 197 p., en allemand.

Heitz C, Luking J, Kempfert HG. 2008. Geosynthetic reinforced and pile supported embankments under static and cyclic loading. In: Proc. of the 4th European Geosynthetics Conf Euro-Geo4, Edinburgh (UK), paper No. 215.

Hewlett WJ, Randolph MF. 1988. Analysis of piled embankment. Ground Eng. 21: 12-18.

Horgan G, Sarsby R. 2002. The arching effect of soils over voids and piles incorporating geosynthetic reinforcement. In: Proc. 7th International Conference on Geosynthetics. Swets and Zeitlinger, Nice, pp. 373-378.

Houda M, Jenck O, Emeriault F. 2016. Physical evidence of the effect of vertical cyclic loading on soil improvement by rigid piles: a small-scale laboratory experiment using Digital Image Correlation. Acta Geotech. 11(2): 325-346, doi: 10.1007/ s11440-014-0350-Z

Huang J, Han J. 2009. 3D coupled mechanical and hydraulic modeling of a geosynthetic reinforced deep mixed column-supported embankment. Geotext. Geomembr. 27: 272-280.

Itasca Consulting Group, Inc. 2009. FLAC3D - Fast Lagrangian Analysis of Continua in Three-Dimensions, Version 4.0, User's guide. Minneapolis: Itasca.

Jenck O. 2005. Le renforcement des sols compressibles par inclusions rigides verticales. Modélisation physique et numérique. Thèse de doctorat, INSA Lyon.

Jenck O, Dias D, Kastner R. 2007. Two-dimensional physical and numerical modeling of a pile-supported earth platform over soft soil. J. Geotech. Geoenviron. Eng. 133: 295-305.

Jenck O, Dias D, Kastner R. 2009. Three-dimensional numerical modelling of a piled embankment. Int. J. Geomech. 9(3): 102-112, doi: 10.1061/(ASCE)1532-3641(2009)9:3(102).

Jenck O, Combe G, Emeriault F, De Pasquale A. 2014. Arching effect in a granular soil subjected to monotonic or cyclic loading: kinematic analysis. In: 8th International Conference on Physical Modelling in Geotechnics, 14-17 January 2014, Perth, Australia, edited by Christophe Gaudin and David White, pp. 1243-1249. doi:10.1201/b16200-179. 
Kempfert HG, Stadel M, Zaeske D. 1997. Berechnung von geokunststoffbewehrten Tragschichten über Pfahlelementen. Bautechnik 74: 818-825.

Laurent Y, Dias D, Simon B, Kastner R. 2003. A 3D finite difference analysis of embnkments over pile-reinforced soft soil. In: Proc. of the Int. Workshop on Geotechnics of Soft Soils - Theory and Practice, 17-19 September 2003, Noordwijkerhout, Pays-Bas. Essen: Verlag Glückauf, pp. 271-276.

Low BK, Tang SK, Choa V. 1994. Arching in piled embankments. J. Geotech. Geoenviron. Eng. 120: 1917-1938.

Magnan JP. 1994. Methods to reduce the settlement of embankments on soft clay: a review. In: Speciality Conference on the Foundations and Embankments Deformations, ASCE (Geotechnical Special Publication No. 40), pp. 77-90.

Morgon M. 2010. Renforcement de sol par inclusions rigides : étude du mécanisme de transfert de charge dans le matelas de répartition. Mémoire du diplôme d'ingénieur, Polytech Clermont-Ferrand, $120 \mathrm{p}$.

Okyay US, Briançon L. 2012. Monitoring and numerical investigations of rigid inclusion reinforced concrete water tank. In: $3 \mathrm{rd}$ International Conference on New Developments in Soil Mechanics and Geotechnical Engineering, 28-30 June 2012, Near East University, Nicosia, North Cyprus.

Okyay US, Dias D. 2010. Use of lime and cement treated soils as pile supported load transfer platform. Eng. Geol. 114: 34-44.
Okyay US, Dias D, Thorel L, Rault G. 2014. Centrifuge modeling of a pile-supported granular earth-platform. J. Geotech. Geoenviron. Eng. 140(2): 04013015-1-04013015-12, doi: 10.1061/(ASCE) GT.1943-5606.0001004.

Rault G, Thorel L. 2009. Étude du transfert de charge par cisaillement. Essais de réception par cisaillement. Dispositif de plateau mobile.

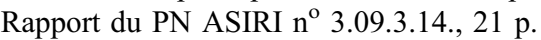

Santruckova H. 2012. Inertial loading of soil reinforced by rigid inclusions to an upper flexible layer. Thèse de doctorat, université de Grenoble.

Thai Son Q, Hassen G, de Buhan P. 2010. Seismic stability analysis of piled embankments: a multiphase approach. Int. J. Numer. Anal. Methods Geomech. 34: 91-110.

Van Eekelen JM, Van MA, Bezuijen A. 2007. The Kyoto Road, a fullscale test. Measurements and calculations. In: 14th European Conference on Soil Mechanics and Geotechnical Engineering, Madrid, Millpress, Rotterdam, pp. 1533-1538.

Van Eekelen SJM, Bezuijen A, Van Tol AF. 2012a. Model experiments on piled embankments. Part I. Geotext. Geomembr. 32: 69-81.

Van Eekelen SJM, Bezuijen A, Van Tol AF. 2012b. Model experiments on piled embankments. Part II. Geotext. Geomembr. 32: 82-94.

Villard P, Grange S. 2013. Influence des cycles de chargement/ déchargement sur les mécanismes de transfert de charges dans les matelas granulaires. Rapport du laboratoire 3SR pour la FNTP.

Citation de l'article : Moustafa Houda, Orianne Jenck, Fabrice Emeriault. Massifs de sol renforcés par inclusions rigides : analyse de l'effet d'un chargement cyclique vertical quasi statique par expérimentation sur modèle réduit et validation numérique. Rev. Fr. Geotech. 2016, 147,2 . 